\title{
Doping of a Plate-Type Acoustic Metamaterial
}

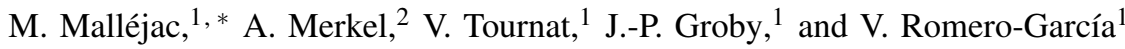 \\ ${ }^{1}$ Laboratoire d'Acoustique de l'Université du Mans (LAUM), UMR 6613, \\ Institut d'Acoustique - Graduate School (IA-GS), CNRS, Le Mans Université, France \\ ${ }^{2}$ Université de Lorraine, CNRS, IJL, F-54000 Nancy, France
}

(Dated: December 18, 2020)

\begin{abstract}
We theoretically, numerically, and experimentally investigate the feasibility of acoustic doping, i.e., changing one of the effective properties of a medium by adding an impurity, to achieve super-squeezing. This effect, characterized by perfect and zero-phase transmission, can be obtained with zero index media. In acoustics, zero-phase propagation can be achieved with a plate-type acoustic metamaterial (PAM) acting as a density-near zero metamaterial (DNZ). We point out the possibility of modifying the compressibility of a DNZ medium by mounting a Helmholtz resonator in parallel with the PAM. We are then able to dope the system and to turn it into a Density and Compressibility Near Zero (DCNZ) medium, thus allowing super-squeezing.
\end{abstract}

The tremendous boom of electromagnetic and acoustic metamaterials [1-4] has opened up a wide range of unexpected applications and design options for wave manipulation and control, including bending, cloaking, focusing, as well as energy trapping. A carefully engineered structure can bring an effective medium to extreme macroscopic parameters, such as a near-zero refractive index $n(\omega)=\sqrt{\mu \varepsilon} \approx 0$ in electromagnetics. Zero-Index media, in which one or more of the constitutive parameters vanish, can be classified into three categories [5, 6]: Epsilon-Near-Zero (ENZ) [7--12] (almost zero permittivity $\varepsilon \approx 0$ ), Mu-Near-Zero (MNZ) [13-15] (almost zero permeability - $\mu \approx 0$ ), or Epsilon and Mu-NearZero media (EMNZ) [16, 17] (both the permittivity and the permeability are zero at a given frequency, $\varepsilon \approx 0, \mu \approx 0$ ). As a result, the wavelength is stretched and gives rise to a staticlike field, a large phase velocity, and thus a nearly constant phase distribution along the material, allowing directivity patterning [18], tunnelling, and supercoupling (full transmission of the incident wave with no distortion or phase change along the material) [19, 20]. It is worth noting that, although there is almost no spatial variation, the field still oscillates in time. The wavelength enlargement is accompanied by a decoupling of the spatial and temporal field variations [6].

EMNZ media have the particularity of being able to satisfy both the zero-phase propagation, associated with the staticlike ENZ field distribution, and the impedance matching condition to free space (impedance $Z_{0}$ ), given for normal incidence by $Z=\sqrt{\mu / \varepsilon}=Z_{0}$. An interesting way to design EMNZ structures is to use doping, i.e., a control of the material macroscopic parameters by locally embedding appropriate inclusions/impurities, in an ENZ metamaterial [21-24]. Liberal et al. [25] showed that the inclusion of a single welldesigned impurity can transform the effective properties of an ENZ body into those of an EMNZ, thus leading to full transmission without phase delay, regardless of the host geometry and doping impurity location. It is important to note here that homogenization remains possible even with a small number of potentially large impurities thanks to the ENZ effective stretching of the wavelength [25].

Equivalent effects to zero-index ones can be found in acoustics by analogy with the transverse magnetic modes in electro- magnetism. The acoustic constitutive parameters analogous to $\varepsilon$ and $\mu$ are respectively the density $\rho$ and the compressibility $C$ (inverse of the bulk modulus $\kappa$ ). Quasistatic field distribution and zero-phase propagation can therefore be achieved using Compressibility and Density-Near-Zero (DCNZ) [36, 37], Compressibility-Near-Zero (CNZ) [26] or Density-Near-Zero (DNZ) media [27-[31]. A periodic arrangement of thin elastic plates (or membranes) in air - a Plate-type Acoustic Metamaterial (PAM) - makes the DNZ condition possible thanks to the strong dispersion around the band gap associated with the plate resonance. Such a system can be characterized with the help of three different frequencies [31]. The impedance matching occurs at the plate resonance frequency but is accompanied by a phase delay depending on the length of the system. At the exact zero effective density frequency, none of the supercoupling condition, i.e., zero-phase propagation and impedance matching, is met as it can be seen with the transmission coefficient (module and phase) of a PAM of thickness $L$, cross-section $S$, effective compressibility $C=C(\omega)$, and density $\rho=\rho(\omega)$,

$$
\begin{aligned}
& |T|=\left(\cos ^{2}(\omega \sqrt{\rho C} L)+\frac{1}{4}\left[\sqrt{\frac{\rho C_{0}}{C \rho_{0}}} \frac{S_{0}}{S}+\sqrt{\frac{\rho_{0} C}{C_{0} \rho}} \frac{S}{S_{0}}\right]^{2} \sin ^{2}(\omega \sqrt{\rho C} L)\right)^{-1 / 2}, \\
& \phi=-\operatorname{atan}\left(\frac{1}{2}\left[\sqrt{\frac{\rho C_{0}}{C \rho_{0}}} \frac{S_{0}}{S}+\sqrt{\frac{\rho_{0} C}{C_{0} \rho}} \frac{S}{S_{0}}\right] \tan (\omega \sqrt{\rho C} L)\right),
\end{aligned}
$$

with subscript.$_{0}$ referring to the surrounding medium. Although the tangent term vanishes when the density tends to zero, the ratio $\sqrt{C / \rho}$ tends towards infinity, resulting in a non-zero constant phase limit when the density tends to zero (details in the Supplemental). Zero-phase propagation occurs in the negative density regime $\left(\rho(\omega)=-C(\omega) \rho_{0} / C_{0}\right)$ with an impedance mismatch. One way to achieve supercoupling with only a PAM is to embed the latter within a waveguide that has a huge cross-sectional difference with the surrounding waveguides $\left(S / S_{0} \rightarrow 0\right)$, to compensate the impedance mismatch [29]. Another way to achieve both perfect transmission and zero-phase propagation, implemented here, is to use doping to turn the PAM, i.e., the DNZ body, into a Density and Compressibility-Near-Zero (DCNZ) metamaterial $(\rho(\omega) \approx 0$, $C(\omega) \approx 0)$. 


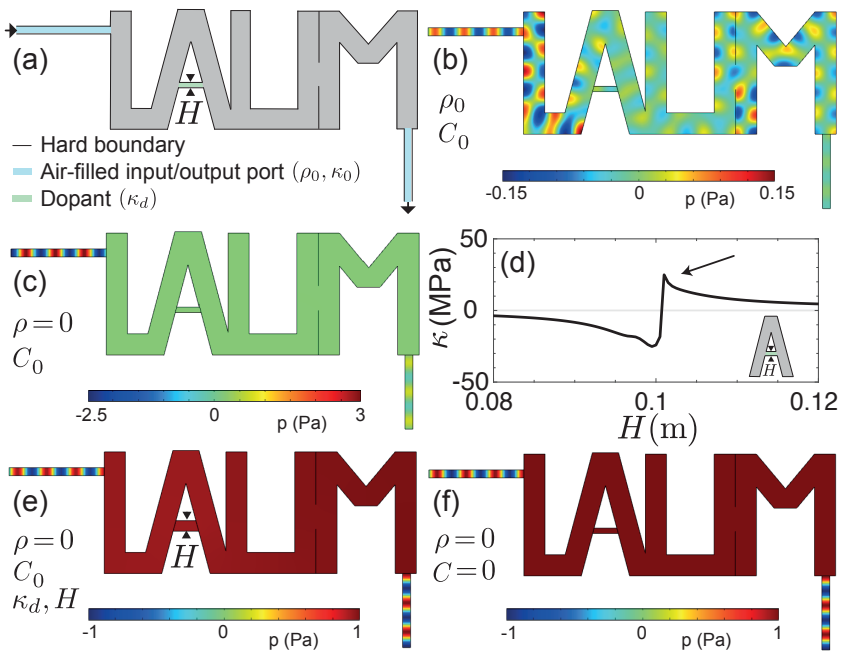

FIG. 1. [Color online] Full-wave simulation of a 2D acoustic doping with a single dopant: sketch of structure (a), pressure field when the medium is filled with air (b), with a DNZ medium (c), with a doped DNZ medium (dopant: transverse bar of the "A" letter, filled with a medium of bulk modulus $\kappa_{d}$ and of width $H$ ) (e) or with a DCNZ medium (f). Figure (d) shows the effective bulk modulus of the entire host "LAUM" depending on the geometry of the dopant $H$. The doping condition to turn the host DNZ medium into an effective DCNZ medium occurs when $C \approx 0$, thus for $\kappa \rightarrow \infty$, at $H=10.1 \mathrm{~cm}$ (arrow in (d)).

In this work, we first investigate the feasibility of an acoustic analogue of the photonic doping effect on a twodimensional DNZ system. Then, we show that a one dimensional lossless PAM can be efficiently doped using a single doping impurity: a tuned Helmholtz resonator. Finally, the influence of both the dopant location and the losses are studied.

We start by a full-wave simulation of the doping phenomenon on a two-port random 2D medium, using the COMSOL multiphysics software. The input and output ports (light blue areas in Fig 11(a)) are air-filled waveguides, plugged into the "L" and "M" shapes respectively. A monochromatic wave of frequency lower than the cut-off frequency of the ports (above which other modes than the plane wave one can propagate) impinges the structure from the left ensuring plane wave propagation. The "LAUM"-shaped medium is filled with air (b), a DNZ medium (c, e), or a DCNZ medium (f). The dimension of the "LAUM" structure (width $L_{x}=3 \mathrm{~m}$ and height $L_{y}=1.15 \mathrm{~m}$ ) is chosen much larger than the acoustic wavelength in the air $\left(\lambda_{0}=27 \mathrm{~cm}\right)$.

Figure 1(b) shows a non-uniform pressure field distribution inside the $2 \mathrm{D}$ medium with high order modes and a weak transmission (7\%). The pressure field is strongly dependent on the geometry of the host. If the host medium is replaced by either a DNZ or a DCNZ medium (Fig. 1.(c) and Fig.11(f)), the pressure field becomes uniform within the medium due to the stretching of the effective acoustic wavelength, allowing a wave propagation without phase delay. In both cases, the pressure field is geometry independent. A tunnelling effect is also achieved when the host medium is a DCNZ (Fig.11f)) in contrast to the case of a DNZ (Fig. 1 ( c)). The host medium is impedance matched to the ports in the DCNZ case, which leads to a zero-phase total transmission.

We propose to reproduce the DCNZ behavior by doping the DNZ host using only a single impurity, i.e., by attributing different medium properties only to a small part of the geometry. We choose here to use the transverse bar of the "A" letter as a dopant. Doping can be achieved by tuning either the bulk modulus $\kappa_{d}$ or the geometry of the dopant. Figure 1]d) shows the dependence of the effective bulk modulus of the whole system on the transverse bar geometry (the width $H$ ) for a fixed value $\kappa_{d}=1.11 .10^{4} \mathrm{~Pa}$. A resonant behavior is observable. The optimal geometry to dope the DNZ host is $H=10.1 \mathrm{~cm}$, that is the width for which the system's effective bulk modulus is the largest, i.e., an almost zero effective compressibility, while the zero effective density remains unchanged. It is worth noting here, that the sensitivity of doping to the dopant geometry directly depends on the variation of the bulk modulus. Sharp variations require a high degree of design precision to achieve a maximum value of $\kappa(\omega)$. Figure 1(e) depicts the pressure field obtained for the doped DNZ host. The system "DNZ \& dopant" exhibits a similar response to that of a medium integrally filled with a DCNZ material, thus evidencing acoustic doping (see videos in the Supplemental).

We now focus on the feasibility to dope a PAM. We consider a $1 \mathrm{D}$ periodic arrangement of $N=20$ thin clamped elastic shims (plastic with a Young's modulus $E=4.6 \mathrm{GPa}$, density $\rho=1400 \mathrm{~kg} \cdot \mathrm{m}^{-3}$, Poisson's ratio $v=0.41$, thickness $h=102 \mu \mathrm{m}$ ) equally spaced by a distance $L_{g a p}=1 \mathrm{~cm}$ and plugged into a waveguide of radius $R_{a}=15 \mathrm{~mm}$. The properties of such a PAM have been extensively studied [29-31]. The detailed transfer matrix model and the analytical acoustic response of the 20-units long PAM can be found in the Supplemental. Impedance matching, zero-density, and zerophase propagation occur at three different frequencies, respectively $f_{m}=422 \mathrm{~Hz}, f_{\rho=0}=414 \mathrm{~Hz}$, and $f_{\phi=0}=405$ $\mathrm{Hz}$. A strong impedance mismatch at the zero-phase propagation frequency prevents such a PAM from total transmission. Doping the PAM results in shifting both the zero-phase and the impedance-matching frequencies towards the zero-density one, since zero-compressibility occurs at the same frequency as the one of zero-density. Therefore, doping should allow to achieve supercoupling effect with a PAM even without a large section change. A doping inclusion mounted in parallel to the waveguide is considered in this system, as it is necessary to act on the effective bulk modulus [32-34]. The chosen dopant is a Helmholtz resonator, which is easily tunable in practice and is of subwavelength dimensions. The neck length $L_{n}=20 \mathrm{~mm}$ and the radii of the neck $R_{n}=2 \mathrm{~mm}$ and of the cavity $R_{c}=10$ $\mathrm{mm}$ are fixed, while the cavity length $L_{c}$ is adjustable with a piston as shown in Fig. 2(a).

We first analyze the lossless case depicted in Fig. 2, where the Helmholtz resonator is mounted between the 10-th and 11-th plates of the PAM. In order to find the configuration where doping occurs, we apply a similar procedure to that 

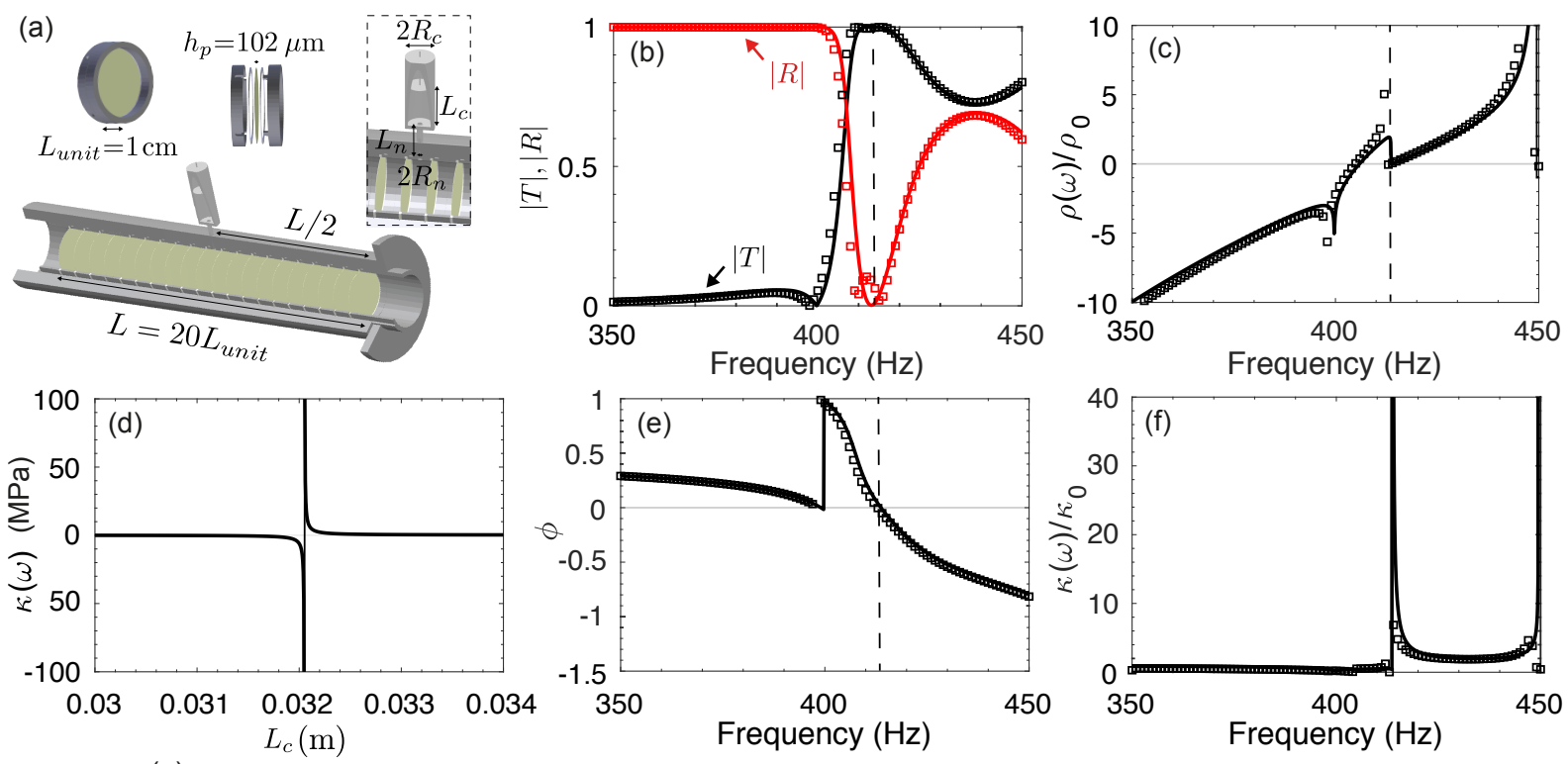

(g)

(h)

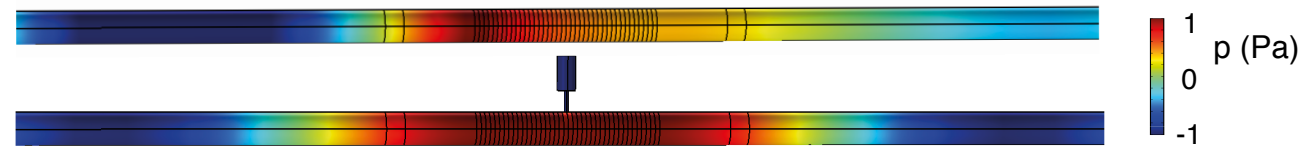

FIG. 2. [Color online] Doping of a 20-units long lossless PAM by adding a Helmholtz resonator: 3D sketches of the unit cell, of the 20 plates metamaterial doped in its middle by a Helmholtz resonator, and of a close-up on the resonator (a). Transmission (black) and reflection (red) magnitudes (b), real part of the system effective density (c), phase of the transmission coefficient (e), and real part of the system effective bulk modulus versus frequency (f). Continuous lines and square symbols represent the analytical and numerical results respectively. Figure (d) shows the dependence of the effective bulk modulus on the Helmholtz cavity length $L_{c}$. Figures $(\mathrm{g})$ and $(\mathrm{h})$ depict the total pressure field of a 20-units long PAM without and with a dopant at the zero-phase propagation frequency $f=405 \mathrm{~Hz}$ and $f=414 \mathrm{~Hz}$ respectively (full-wave simulation).

in Fig. 1.d), i.e., we look for the configuration that produces a maximum value of effective bulk modulus. This optimal configuration corresponds to a length $L_{c}=32.06 \mathrm{~mm}$ and requires to be dimensioned with an extreme precision. Figures 2 (b,c,e,f) show respectively the amplitude of the scattering parameters, the effective dynamic mass density, the phase of the transmission coefficient and the effective bulk modulus for the configuration mentioned above. The analytical results are validated against those from a 3 dimensional fullwave simulation shown by the square symbols in Fig. 2. A zero-density accompanied by a maximum of bulk modulus is found at $f=414 \mathrm{~Hz}$. At this particular frequency, the total system behaves as a DCNZ metamaterial as evidenced by the scattering parameters. The zero value of the transmission phase occurs with a zero reflection and an unitary transmission, i.e., zero-phase propagation and impedance matching are combined. We thus confirm the possibility to realize supercoupling with a PAM using doping.

Figures $2(\mathrm{~g}, \mathrm{~h})$ show the total pressure field, respectively without and with the doping impurity, to illustrate the impact of the dopant at the zero-phase frequency. In both cases, we observe a zero-phase propagation either with an impedance mistmatch or with a full transmission. In the latter case, the pressure field is perfectly symmetric with respect to the PAM,

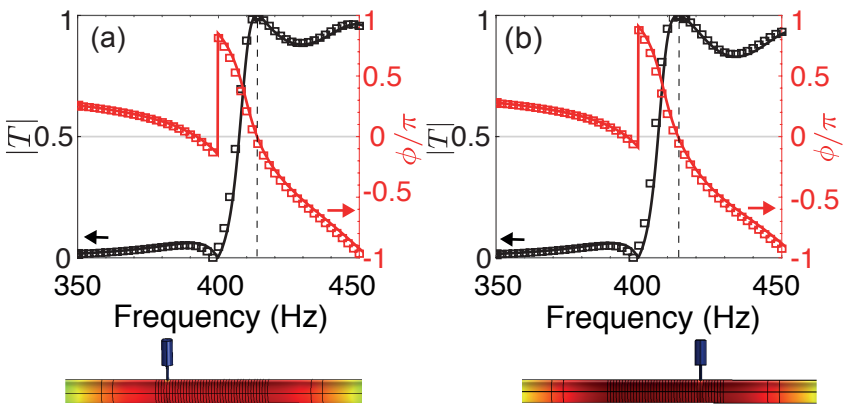

FIG. 3. [Color online] Influence of the dopant position: transmission magnitude (black), phase of the transmission coefficient (red), and pressure field along the metamaterial. Tuned Helmholtz resonator placed between the 2nd and 3rd plates (a) or between the 15-th and 16-th plates (b). Continuous lines and square symbols represent the analytical and numerical results respectively.

which is characteristic of the supercoupling effect. In contrast, although zero-phase propagation is satisfied when doping is not reached, the pressure field is not symmetric (see Fig. 22 (g)) due to the non-unitary transmission (impedance mistmatch).

Another property of interest of DCNZ doping is its independence from the dopant location. Whatever the Helmholtz resonator location in the PAM, doping occurs. To illustrate 
this property, Fig. 3 shows two examples with different locations of the Helmholtz resonator. The dopant is mounted between the 2nd and 3rd plates in the first case, Fig. 3(a), while it is placed between the 15-th and the 16-th in the second, Fig. 3. b). Zero-phase total transmission is observed in both configurations at $f=414 \mathrm{~Hz}$. The independence on the location is due to the large wavelength in the PAM and allows a high freedom in the design of the system.

We now analyze the robustness of doping in the presence of losses. The full lossy problem is solved with both the viscothermal losses in the main waveguide as well as in the Helmholtz resonator and the viscoelastic losses in the plates. Viscothermal losses (mainly due to friction on the walls of the waveguide) are accounted for by considering complex and frequency dependent impedance and wavenumber $Z_{0}(\omega)$ and $k_{0}(\omega)$ respectively [35] (see Supplemental), while the viscoelastic losses are modeled by adding an imaginary part to the plate's Young modulus $E=E_{0}(1+i \beta)$.

Visco-elasticity being the dominant source of losses (see Supplemental), the number of plates in the PAM is reduced to 6. In doing so (see Fig. 4(b-c)), the transmission of the PAM at the zero-phase frequency $f_{\phi=0}=390 \mathrm{~Hz}$ is $\left|T_{f_{\phi=0}}\right|=0.47$ (0.42 measured in 31). A large frequency offset separates this zero-phase frequency from the zero-density and impedance matched frequencies, respectively occurring at $f_{\rho=0}=414$ $\mathrm{Hz}$ and $f_{m}=439 \mathrm{~Hz}$. To confirm the analytical and numerical simulations, the scattering of the real doped system is also measured using a 4 microphones impedance tube (see Supplemental). In the following, solid lines, dashed lines and circle symbols represent the analytical, numerical, and experimental results respectively, the agreement of which is found to be very good.

The inset in Fig. 4(f) shows the evolution of the effective lossy bulk modulus with respect to the length of the Helmholtz resonator cavity. A maximum of real part of the bulk modulus is obtained for the optimal length $L_{c}=36.75 \mathrm{~mm}$. It is worth noting here that the value of this maximum is much lower (by a factor $10^{3}$ ) than in the previously presented lossless case. Figures 4 (d-f) give the response of the lossy system using this optimal configuration. The zero-phase frequency is up shifted to $f_{\phi=0}=412 \mathrm{~Hz}$ and gets closer to the zero-density frequency of the system, i.e., $f_{\rho=0}=414 \mathrm{~Hz}$.

The careful design of the dopant thus allows to strongly reduce the frequency offset between zero-phase, maximum of transmission and zero-density frequencies. As a result the zero-phase propagation frequency $f_{\phi=0}$ gets closer to $f_{m}$. In contrast to the lossless case, the losses prevent from a perfect coincidence of maximal transmission and zero-phase propagation frequencies. As such, losses that are inherently present in any acoustic system can clearly limit the effectiveness of doping. Nonetheless, it is worth noting here that the doping condition allows to have a zero-phase propagation with a $13 \%$ higher transmission (according to the analytics and numerics, and $40 \%$ according to the measurements), the measured (resp. analytical and numerical) magnitude of which goes from 0.42 (resp. 0.47) without dopant to 0.59 (resp. 0.53) in the doped

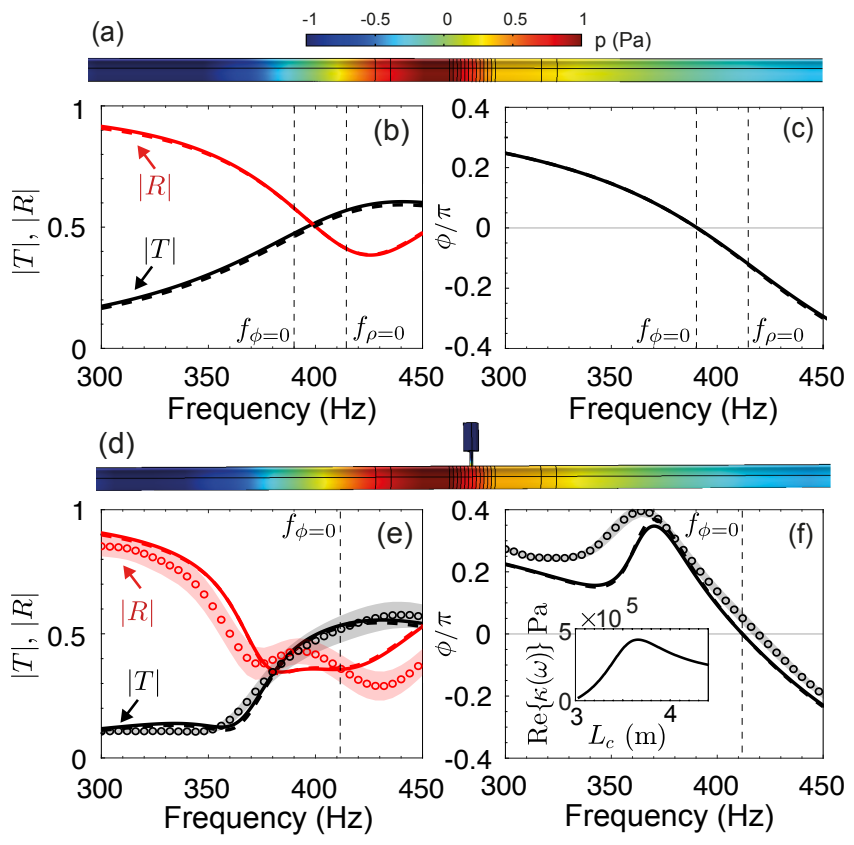

FIG. 4. [Color online] Doping in presence of losses: (a,d) pressure field (full-wave simulation), (b,e) reflection (red) and transmission (black) magnitude, and (c,f) phase of the transmission coefficient of a 6-units long lossy PAM (a-c) and a 6-units long lossy PAM doped with a Helmholtz resonator $\left(L_{c}=36.75 \mathrm{~mm}, R_{c}=10 \mathrm{~mm}, L_{n}=20\right.$ $\mathrm{mm}$, and $R_{n}=2 \mathrm{~mm}$ ) mounted between plates 3 and 4 (d-f). Inset in (f) depicts the geometry optimization on the cavity length of the dopant. Continuous lines and dashed lines represent the analytical and numerical results. The experimental data are presented in a statistical form, with the symbols being the average of the 23 observations (system disassembled then reassembled) and the colored area the statistical standard error, i.e., the std around the mean value.

configuration. Reducing the losses, i.e., finding plates with lower viscoelastic losses, would lead to a better efficiency of the process.

Conclusions. In this study, we reported on the feasibility of doping acoustic structures using only one impurity and we highlighted the limitations induced by the viscothermal and viscoelastic losses. We have demonstrated that the integration of a carefully designed element into a DNZ metamaterial can modify the effective compressibility of the structure so that the system responds as a DCNZ metamaterial at the exact zero density frequency of the non-doped system. The dopant can be designed based on either its bulk modulus value or its geometry. In doing so, a perfect transmission can be achieved in addition to the zero-phase propagation, induced by the stretching of the effective wavelength, thus meeting the requirements of tunnelling and supercoupling for the lossless case. Moreover, the DCNZ condition is achieved independently of both the host geometry and the dopant location. Doping is therefore a good alternative to the large change in cross-section required to observe supercoupling with a PAM, since it requires only one element to be added to the system. The presence of losses, mainly the plate viscoelastic ones for a PAM, has 
an impact on the ability to obtain zero effective compressibility, i.e., to reach an infinite effective bulk modulus. The phenomenon of doping, although still present, is therefore limited and depends on the number of plates in the system. In the considered case of a 6-units long PAM, doping significantly reduces the frequency offset between the zero-phase, zero-density and maximum of transmission frequencies. As a result, the transmission amplitude of the zero-phase wave is increased by almost $15 \%$ compared to the non-doped system. The choice of plates with lower losses would increase the effectiveness of doping. Although complex geometries with sharp angles may restrict the use of PAMs, the doping phenomenon can be applied to any other DNZ systems, thus enabling a high freedom in the design as well as the filling of more complex geometries such as the 2D "LAUM" shape.

This article is based upon work from COST Action DENORMS CA15125, supported by COST (European Cooperation in Science and Technology). This work was funded by the Metaroom Project No. ANR-18-CE08-0021 and co-funded by ANR and RCG.

* matthieu.mallejac@univ-lemans.fr

[1] N. Engheta and R. W. Ziolkowski, Metamaterials : physics and engineering explorations (WileyInterscience, 2006).

[2] K. Sakoda, Electromagnetic metamaterials: modern insights into m (Springer, 2019).

[3] V. Romero-García and A. Hladky-Hennion, Fundamentals and Applications of Acoustic Metamaterials (John Wiley \& Sons, Ltd, 2019).

[4] F. Zangeneh-Nejad and R. Fleury, "Active times for acoustic metamaterials," Reviews in Physics 4, 100031 (2019)

[5] N. Engheta, "Pursuing near-zero response,"'Science 340, 286287 (2013)

[6] I. Liberal and N. Engheta, "Near-zero refractive index photonics," Nature Photonics 11, 149-158 (2017)

[7] M. G. Silveirinha, A. Alú, B. Edwards, and N. Engheta, "Overview of theory and applications of epsilon-near-zero materials," in URSI General Assembly (2008).

[8] B. Edwards, A. Alù, M. E. Young, M. Silveirinha, and N. Engheta, "Experimental verification of epsilon-near-zero metamaterial coupling and energy squeezing using a microwave waveguide," Physical Review Letter 100, 033903 (2008).

[9] A. Alù and N. Engheta, "Dielectric sensing in $\varepsilon$-near-zero narrow waveguide channels," Physical Review B 78, 045102 (2008)

[10] A. Alù, M. G. Silveirinha, and N. Engheta, "Transmissionline analysis of $\varepsilon$-near-zero-filled narrow channels," Physical Review E - Statistical, Nonlinear, and Soft Matter Physics 78, 016604 (2008)

[11] A. Alù and N. Engheta, "Coaxial-to-waveguide matching with $\varepsilon$-near-zero ultranarrow channels and bends," IEEE Transactions on Antennas and Propagation 58, 328-339 (2010).

[12] M. H. Javani and M. I. Stockman, "Real and imaginary properties of epsilon-near-zero materials," Physical Review Letter 117, 107404 (2016)

[13] J. S. Marcos, M. G. Silveirinha, and N. Engheta, " $\mu$-near-zero supercoupling," Physical Review B 91, 195112 (2015)
[14] H. Feng Ma, J. Hui Shi, Q. Cheng, and T. Jun Cui, "Experimental verification of supercoupling and cloaking using mu-nearzero materials based on a waveguide," Applied Physics Letters $103(2013)$

[15] G. Lipworth, J. Ensworth, K. Seetharam, J. S. Lee, P. Schmalenberg, T. Nomura, M. S. Reynolds, D. R. Smith, and Y. Urzhumov, "Quasi-static magnetic field shielding using longitudinal mu-near-zero metamaterials," Scientific Reports 5, 12764 (2015)

[16] A. M. Mahmoud and N. Engheta, "Wave-matter interactions in epsilon-and-mu-near-zero structures," Nature Communications 5, $5638(2014)$

[17] J. J. Yang, Y. Francescato, S. A. Maier, F. Mao, and M. Huang, "Mu and epsilon near zero metamaterials for perfect coherence and new antenna designs," Opt. Express 22, 9107-9114 (2014)

[18] A. Alù, M. G. Silveirinha, A. Salandrino, and N. Engheta, "Epsilon-near-zero metamaterials and electromagnetic sources: Tailoring the radiation phase pattern," Physical Review B 75, 155410 (2007)

[19] A. Alù and N. Engheta, "Light squeezing through arbitrarily shaped plasmonic channels and sharp bends," Physical Review B 78, 035440 (2008)

[20] B. Edwards, A. Alù, M. G. Silveirinha, and N. Engheta, "Reflectionless sharp bends and corners in waveguides using epsilon-near-zero effects," Journal of Applied Physics 105, 044905 (2009)

[21] M. Silveirinha and N. Engheta, "Design of matched zero-index metamaterials using nonmagnetic inclusions in epsilon-nearzero media,"'Physical Review B 75, 075119 (2007).

[22] I. Liberal, Y. Li, and N. Engheta, "Reconfigurable epsilonnear-zero metasurfaces via photonic doping," Nanophotonics 7,

[23] E. Nahvi, I. Liberal, and N. Engheta, "Nonperturbative effective magnetic nonlinearity in enz media doped with kerr dielectric inclusions," ACS Photonics 6, 2823-2831 (2019).

[24] Z. Zhou, Y. Li, H. Li, W. Sun, I. Liberal, and N. Engheta, "Substrate-integrated photonic doping for near-zero-index devices," Nature Communications 10, 4132 (2019)

[25] I. Liberal, A. M. Mahmoud, Y. Li, B. Edwards, and N. Engheta, "Photonic doping of epsilon-near-zero media," Science 355, 1058-1062 (2017).

[26] H. Esfahlani, M. S. Byrne, M. McDermott, and A. Alù, "Acoustic supercoupling in a zero-compressibility waveguide," Research 2019, 1-10 (2019)

[27] Z. Yang, J. Mei, M. Yang, N. H. Chan, and P. Sheng, "Membrane-type acoustic metamaterial with negative dynamic mass," Physical Review Letters 101, 204301 (2008).

[28] R. Graciá-Salgado, V. M. García-Chocano, D. Torrent, and J. Sánchez-Dehesa, "Negative mass density and $\rho$-near-zero quasi-two-dimensional metamaterials: Design and applications,"'Physical Review B 88, 224305 (2013),

[29] R. Fleury and A. Alù, "Extraordinary sound transmission through density-near-zero ultranarrow channels," Physical Review Letters 111, 055501 (2013)

[30] F. Bongard, H. Lissek, and J. R. Mosig, "Acoustic transmission line metamaterial with negative/zero/positive refractive index," Physical Review B 82, 094306 (2010)

[31] M. Malléjac, A. Merkel, J. Sánchez-Dehesa, J. Christensen, V. Tournat, J.-P. Groby, and V. Romero-García, "Zero-phase propagation in realistic plate-type acoustic metamaterials," Applied Physics Letters 115, 134101 (2019)

[32] N. Cselyuszka, M. Sečujski, and V. C. Bengin, "Compressibility-near-zero acoustic metamaterial," Physics Letters A 378, 1153 - 1156 (2014) 
[33] N. Fang, D. Xi, J. Xu, M. Ambati, W. Srituravanich, C. Sun, and X. Zhang, "Ultrasonic metamaterials with negative modulus," Nature Materials 5, 452-456 (2006)

[34] S. H. Lee, C. M. Park, Y. M. Seo, Z. G. Wang, and C. K. Kim, "Composite acoustic medium with simultaneously negative density and modulus," Physical Review Letter 104, 054301 (2010)

[35] C. Zwikker and C. W. Kosten, "Sound absorbing materials,"
(1949).

[36] C. Xu, G. Ma, Z.-G.. Chen, J. Luo, J. Shi, Y. Lai, and Y.. Wu, "Three-dimensional acoustic Double-Zero-Index Medium with a fourfold degenerate Dirac-like Point," Physical Review Letters 124, $074501(2020)$

[37] M. Dubois, C. Shi, X. Zhu, Y. Wang, and X. Zhang, "Observation of acoustic Dirac-like cone and double zero refractive index," Nature Communications 8, 14871 (2017) 